\title{
La educación ambiental rural desde las escuelas básicas y por estas
}

\section{Rural Environmental Teaching in Basic General Education}

\author{
Martha Isabel Martínez Pacheco' \\ Escuela Normal María Mazzarello \\ Masaya, Nicaragua \\ martha210467@gmail.com \\ Lisa Carballo Carrillo \\ Ministerio de Educación \\ Orange Walk Town, Belize \\ lisa.carrillo95@gmail.com
}

\begin{abstract}
Recibido 28 de mayo de 2012 • Corregido 08 de marzo de 2013 • Aceptado 13 de marzo de 2013
Resumen. En el presente artículo, basado en los contextos nicaragüenses y beliceños, abordamos la necesidad de una educación ambiental del estudiantado de primaria, para garantizar que esta generación joven viva en armonía con la naturaleza, lo cual dependerá de sus prácticas sanas para proteger el planeta. Pretendemos, entonces, despertar el interés del personal docente para que, de una forma organizada, planificada y consciente, construya estrategias pedagógicas ambientalistas, con el propósito de lograr que sus estudiantes tengan una conciencia fortalecida en amor a su cultura y a su comunidad, y que aspiren a vivir en hogares limpios, para, así, prosperar en convivencia, en cultura material, espiritual, en economía y en desarrollo.
\end{abstract}

Palabras claves. Educación ambiental, desarrollo sustentable y participación comunitaria, Nicaragua, Belice, Revista Electrónica Educare.

Abstract. This paper, based on the Nicaraguan and Belizean contexts, points out the need of providing environmental education to students from the $1^{\text {st }}$ to $6^{\text {th }}$ grades of the Basic General Education to ensure that young generations will live in harmony with nature, which will depend on their healthy practices to protect the planet. For this, we attempt to stimulate the interest of teachers to create environmental teaching/learning strategies in an organized, planned, conscious way to raise students' awareness and love for their culture and community, and for students to aspire to live in healthy homes. This way, students will succeed in the coexistence experience, in the material and spiritual cultures, and in the economic and development areas.

Keywords. Environmental education, sustainable development, community participation, Nicaragua, Belize

1 Master en Educación Rural Centroamericana. Actualmente es asesora en pedagógica del Ministerio de Educación, Nicaragua.

2 Master en Educación Rural Centroamericana. Actualmente es supervisora curricular en el Ministerio de Educación, Belize C.A. 
URL: http://www.una.ac.cr/educare

CORREO: educare@una.cr

Entre los objetivos del desarrollo sostenible está el de aprovechar y restaurar los recursos naturales para evitar, a toda costa, la degradación de los recursos, proteger la capacidad límite de la naturaleza, favorecer la restauración y evitar los efectos adversos sobre la calidad del aire, agua y tierra, con el fin de perpetuar la oferta ambiental de los ecosistemas (Solís, Madrigal, Borrsé y Coto, 1998).

La educación ambiental de los niños, niñas y adolescentes es la garantía para lograr un desarrollo sustentable.

Conformando esta meta y teniendo como derecho digno un ambiente sano y equilibrado, entendemos la importancia de la educación ambiental como la herramienta para lograr nuestros objetivos de formar individuos ecologistas responsables y respetuosos. De esta manera, esperamos incorporar una educación para la vida porque ha de tejer nuestra identidad, cultura, forma de vivir, costumbres y prácticas, con la intención de formar ciudadanos amantes de la naturaleza y comprometidos con su cuidado, como fuente de un futuro sano; esto es, participantes de un desarrollo sustentable.

Poco haremos si los docentes no están dispuestos a actualizar sus saberes. Es necesario que se documenten y reflexionen entorno a cómo estamos viviendo, qué esta pasando en el mundo, qué acciones hacemos que destruyen el planeta. Luego pasar al otro plano: Cómo he estado formando a mis estudiantes para que vivan en fraternidad con la naturaleza? ¿Han alcanzado las competencias de vivencias sanas? ¿Qué más puedo hacer o que debo cambiar en mis intervenciones pedagógicas?

En esta faena no basta que el personal docente se autoevalúe e inicie el proceso transformador: es importante que todos los sectores sociales y organizaciones le acompañen y unan esfuerzos.

Sin una participación dinámica e integral de los sectores sociales que conviven en una localidad, no se podrá despertar en la población estudiantil una conciencia que le permita identificarse con la problemática ambiental de su comunidad, de su país y del mundo. Por eso todos los sectores deben involucrarse y estar en la misma sintonía, pues el interés y el bien es común.

\section{Conceptos, enfoques pedagógicos y participación comunitaria}

\section{¿Qué es la educación ambiental rural?}

La tierra no es herencia de nuestros padres sino préstamo de nuestros hijos.

Pensamiento indoamericano

La educación ambiental es más que solo compartir información, es una forma de vivir.

Ortega y Velasco (2006) explican que en la educación ambiental "el individuo adquiere conocimientos, interioriza actitudes y desarrolla hábitos que le permiten modificar la conducta individual y colectiva en relación con el ambiente en el que se desenvuelve" (...) (p. 13). 
Es decir, el proceso de acción-reflexión-acción ocurre para alcanzar una mejor vida en armonía con la identidad, cultura, sociedad, comunidad, persona... en fin, con todo su contexto. Como parte del entorno rural, se hace necesario integrar y desarrollar al tejido rural, el concepto de educación ambiental rural.

Del proyecto, Educación participativa sobre la gente y la naturaleza (Solís et al. 1998) podemos rescatar que hay que orientar nuestros intereses a procesos creativos que potencien las fortalezas con las comunidades cercanas a las áreas protegidas y con estas. Una educación participativa para la conservación de los recursos naturales.

La educación ambiental rural orientada en el "currículo nacional básico" de Nicaragua (División General de Currículo y Desarrollo Tecnológico. Managua, Nicaragua, 2009) tiene como objetivo transformar las prácticas educativas para lograr que los estudiantes desarrollen competencias que les serán útiles para la vida, el trabajo y la convivencia. Se comprende que el accionar ambiental debe ir más allá de la teoría, más allá de una clase de ciencias naturales, es decir, llegar a la familia y a la comunidad. Veamos algunos aspectos enfocados en un proceso que atienda la nueva ruralidad:

- Visión integral de la realidad: Fomenta la visión integral e integrada de la realidad, con base en su análisis pluri interdisciplinario, que permite estudiarla desde diversos ángulos, y contribuir, así, a la integración del conocimiento de manera sistémica, visto como un todo armónico en el aprendizaje de los grupos estudiantiles.

- Desarrollo de potencialidades: Constituye un proceso continuo que desarrolla las potencialidades del ser humano en las dimensiones de aprender a aprender, aprender a ser, aprender a hacer, aprender a emprender y aprender a convivir.

- Identidad personal, nacional y centroamericana: Enfatiza el desarrollo de las estructuras y procesos intelectuales, los valores personales y socialmente positivos, la búsqueda y consolidación de la identidad personal, comunitaria, nacional y centroamericana. La formación de una personalidad sana y creativa tiene la capacidad de abrirse a otras culturas, al conocer y valorar la interculturalidad, las costumbres, tradiciones de la comunidad, región y país. En este sentido, contribuye a crear y fortalecer la identidad nacional y centroamericana. (...). (División General de Currículo y Desarrollo Tecnológico. Managua, Nicaragua, 2009, p. 23)

- Diversidad: Incorpora la diversidad de necesidades, intereses, problemas y potencialidades de los estudiantes, familia y comunidad, como fuente para el desarrollo del currículo y para el diseño de situaciones de aprendizaje socialmente útiles en términos de una educación para la vida. Integra, además, la atención a las personas con necesidades educativas especiales con discapacidad o sin esta. El respeto a la diversidad fundamenta la convivencia social, la democracia y la equidad en todas sus 
URL: http://www.una.ac.cr/educare

CORREO: educare@una.cr

dimensiones (social, económica, política, cultural, ambiental, generacional y territorial. (...). (División General de Currículo y Desarrollo Tecnológico. Managua, Nicaragua, 2009, p. 22)

- Interacción, participación y descentralización: Principios fundamentales en donde interactúan todos los niveles de la institución, así como los actores sociales beneficiarios de los procesos enseñanza- aprendizaje. Es un proceso participativo de toda la ciudadanía siendo parte integrante de la contextualización del currículo a [sic] Nivel local.

- Interacción: Promueve, dentro de la comunidad educativa, la interrelación entre actores, recursos y procesos del currículo, posibilitando una acción organizada y creadora en las actividades educativas $\rrbracket$

- Participación: Concreta el concepto de comunidad educativa ampliada, fortalecida por la comunidad local, organismos gubernamentales, no gubernamentales, entes autónomos, empresas privadas y demás agentes sociales.

- Descentralización: Introduce, en la gestión educativa nacional, el modelo de planificación curricular estratégico, contextualizado, con esquemas más flexibles, que suponen el desarrollo, a través de la educación de las capacidades locales, para identificar problemas específicos, diseñar soluciones viables, implementarlas y evaluar sus resultados. (División General de Currículo y Desarrollo Tecnológico. Managua, Nicaragua, 2009, pp. 23-24)

A toda esta búsqueda de conciencia y de cultura ambiental nos remite (Gadotti, 2001) en su artículo Pedagogía de la tierra, al proponer para los debates docentes las interrogantes que promueven la reflexión, como agentes trasformadores y formadores. Sugiere que de forma responsable nos debemos preguntar: ¿Qué estamos estudiando en las escuelas? ¿No estaremos construyendo una ciencia y una cultura que sirven para la degradación del planeta y de los seres humanos? Estos temas de discusión, como parte de la reflexión docente, conducen al fin de que visualicen la tierra como un nuevo paradigma, donde los seres humanos se preocupen no solo de su comunidad, su país y el mundo, en un actuar responsable que, abonando a la sustentabilidad planetaria y por ende al desarrollo, tendría como consecuencia el bienestar de todos.

Los valores se pondrán de manifiesto en la equidad y participación en la distribución de tareas, en el compartir de responsabilidades. Se debe comenzar por los adultos para llegar a los educandos. Nuestra meta es lograr que cada individuo adquiera conciencia planetaria y que, en ello, demuestre solidaridad, amor a la naturaleza, amor a sí mismo y amor a todo ser vivo. 


\section{El papel de la escuela rural en la educación ambiental}

En forma integral las escuelas rurales deben aterrizar los ejes transversales propuestos por los sistemas educativos, puesto que en ellos están presentes los objetivos de una formación para la vida, que repercute en la vivencia comunitaria rural.
El mundo es la suma total de nuestras posibilidades vitales.
Jose Ortega y Gasset

El desarrollo rural está ligado a conocimiento y conservación de la biodiversidad, bases para superar la pobreza a la que hemos sido llevados como producto de la dependencia del capitalismo, el cual origina un consumismo indiscriminado, explotación y destrucción de nuestros recursos naturales, de nuestras costumbres ancestrales sanas.

Citamos a continuación los ejes transversales que fortalecen los currículos en los sistemas educativos, que avizoran el enrumbe de la nueva educación, la cual se acerca a la propuesta con su eco pedagogía y su efecto eco formativo en el diario vivir de los estudiantes y las estudiantes (Gadotti, 2001).

- Promover acciones de sensibilización para la protección, conservación y preservación del medio ambiente y los recursos naturales, en el hogar, escuela y comunidad para alcanzar un desarrollo sostenible.

- Poner en práctica una cultura productiva, protectora de los animales.

- Mostrar actitudes de respeto hacia sí mismo y hacia las demás personas, lo cual permite reafirmar la autoestima sin afectar la de otros y otras.

- Mantener una actitud perseverante en los planes, proyectos y distintas actividades en las que participe. Resuelva problemas con capacidad de iniciativa y sentido de responsabilidad.

- Participar en acciones de protección y reforestación del medio ambiente escolar, familiar y comunitario.

Por otra parte, si la educación ambiental va a impactar un cambio en el día a día de nuestras comunidades rurales, entonces debería involucrar a todos y todas, ser práctica, de largo plazo y con un enfoque holístico. También debe tomarse en cuenta a las personas y las comunidades como

Sembremos árboles en la tierra y sembremos amor en nuestro corazón

Marco Antonio Solis principales agentes de lucha por ese cambio sostenible. Un cambio para un futuro mejor y el buen vivir. 
URL: http://www.una.ac.cr/educare

CORREO: educare@una.cr

En la lucha por el logro de este objetivo retomamos los estándares que están promoviendo las instituciones gubernamentales para que la educación ambiental sea relevante y de buen provecho. De la agenda de Belize (Audubon, 2008) se adaptan los siguientes estándares sugeridos:

1. La educación ambiental rural debe involucrar a todos y todas. La participación y la concientización de las escuelas rurales no puede trabajar sin la ayuda de la comunidad, ya sea las otras instituciones, las iglesias, los líderes comunitarios, grupos comunitarios, el medio de comunicación, el gobierno, las ONG. Para lograr la implementación de cualquier programa en este ámbito se necesita de cada uno de las personas trabajando en solidaridad.

2. La educación ambiental rural debe ser parte de un día a día. Para lograr beneficios a largo plazo es importante que nuestras acciones y comportamientos sean vistos como parte de cada uno de nosotros. Este movimiento pudiera resultar en una concientización integral hacia la madre tierra y el convivir de las actividades del día a día. Es lograr que el conocimiento se haga vivo en el diario vivir, así como la escritura o la lectura en la que el ser humano no se separa de ella, diseñar escenarios de aprendizaje que lo ubiquen en situaciones diversas, y no como pasos a seguir en una receta de cocina.

3. La educación ambiental rural debe tomar el enfoque holístico. En esta época de nuevas creaciones y expectativas, es importante ofrecer muchas oportunidades para conocer aspectos culturales, sociales, tecnológicos, científicos y éticos. De esta manera, conectar y entender, en forma abierta, todos los aspectos y hacer decisiones compartidas. Todo esto tuviera que ponerse en consideración para luego apreciar la importancia de cuidar nuestro ambiente.

4. La educación ambiental rural debe ser práctica. No solamente se debe quedar en el discurso, sino en las acciones. Para ser más práctico debe haber personas capaces y con experiencias desde las necesidades locales. Una educación ambiental práctica no solo atiende las necesidades propias, sino contextualiza estas experiencias y acciones hacia un desarrollo sostenible. La formación del estudiante no solo debe quedar en las aulas y durante un tiempo determinado: debe ir más allá de recibir información, de realizar analogías, de exponer lo que sabe, de dar respuestas a cuestionarios o de la reproducción mecánica. El docente debe planificar acciones que prevalezcan en la práctica diaria, proporcionar la oportunidad para que el estudiantado asuma desafíos crecientes y compromisos conscientes de una actitud responsable con su entorno.

5. La educación ambiental rural debe estar en armonía con nuestra madre tierra. Hay que tomar la educación ambiental como una herramienta más para participar en el convivir y el apropiarse del ambiente como parte nuestro. Este amor y aprecio ha de traer una relación y comunicación mutua entre el ambiente y quienes lo habitamos. 


\section{Contexto actual de las prácticas de educación ambiental en escuelas rurales de primaria básica}

Recopilando la discusión de compañeros y compañeras de esta III promoción de la Maestría en Educación Rural Centroamericana, comprendimos que nuestra región tiene, como un reto importante, la educación para la sustentabilidad y el equilibrio ambiental. Claro que la formación de docentes de calidad para las zonas rurales sería la llave para abrir puertas en este camino. Por ende, las prácticas desarrolladas en las comunidades educativas deben ser coherentes con los discursos de igualdad, respeto hacia la vida y el medio ambiente. También es importante conocer y entender cómo trabajan los ecosistemas y el impacto que esto puede despertar, lo cual requiere profundizar en el conocimiento ecológico.

En cuanto a nuestras metodologías, las iniciativas enfocan un cambio de conducta desde el entorno de la persona, puesto que los comportamientos y desempeños de las estudiantes y los estudiantes no evidencian la relación entre lo que aprenden en la escuela y su vida diaria; no consideran el propósito y los usos de la construcción del conocimiento. En este nivel, los prácticas no muestran señales en sus actitudes ante la problemática ambiental.

El uso insuficiente e ineficiente de nuestros pocos recursos no facilita el empuje de muchas fortalezas que ofrecen diferentes actores presentes en la comunidad, como las ONG, las instituciones ambientalistas del Estado y alcaldía, las que también tienen parte en esta responsabilidad y cuentan con un presupuesto, el cual unido a los del Ministerio de Educación resolverían muchas necesidades como capacitaciones, dotación de materiales, etc.

La pobreza y la falta de materiales en las que viven las comunidades rurales no constituyen justificación para evadir la educación ambiental; se debe valorar la existencia de docentes que buscan una salida a la necesidad o necesidades: ser proactivo debe ser una cualidad inherente en el personal docente, como parte de sus competencias, sus capacidades y su eco pedagogía.

Pasando a otro factor de análisis, están las disciplinas de estudio de los programas de educación primaria de Nicaragua. Se ofrecen clases exclusivas para la agricultura, llamadas Orientación técnica vocacional con actividades alusivas al trabajo de la tierra como la agricultura, la importancia de los árboles, cómo hacer semilleros, las herramientas agrícolas y medidas de seguridad, las labores culturales, la limpieza de hierba en la escuela, las plagas y uso de insecticidas Está también la disciplina de ciencias naturales. Pero, ¿qué nos dice la realidad? Los estudiantes, continúan consumiendo productos chatarra, los huertos se secan por falta de cuido, los malos hábitos de higiene son notorios en la basura que se deja al caminar por las calles o en el mismo patio de la escuela. ¿Qué hace falta? ¿Será que los adultos no hemos dado el ejemplo a los pequeños?

Belice presenta un panorama diferente. El docente toma como propia la iniciativa de hacer actividades donde se involucra al estudiantado en el trabajo de tierra, pero las actividades que se realizan en las escuelas rurales no siempre son pertinentes o en otros casos no atendidas. Esto significa que hay mucho que hacer en atender esta necesidad. Las escuelas secundarias 
URL: http://www.una.ac.cr/educare

CORREO: educare@una.cr

en Belice se acercan a estos currículos vocacionales y técnicos; pero las escuelas primarias muy poco. Una educación rural con un enfoque eco pedagógico servirá como el puente hacia la armonía con la tierra.

En la práctica, los docentes nicaragüenses y beliceños abordan estos contenidos de forma teórica y realizan algunas labores de limpieza de la maleza y huertos escolares; no obstante estas actividades están sujetas al periodo dosificado. Una vez concluido el periodo, los estudiantes abandonan los huertos y todo el trabajo realizado se pierde nuevamente. La sustentabilidad se pone en cuestión. ¿Por qué no consumir en la escuela las verduras cosechadas, hacer ferias alimenticias, convivencias familiares? ¿Por qué no ir al parque cercano a sembrar árboles? ¿Por qué no hacer charlas a los padres de familia directamente en sus hogares? ¿Será considerado más trabajo hacer práctico el currículo?

En los esfuerzos por transformar los hábitos de los estudiantes de primaria, también se unen diferentes instituciones y organizaciones cooperantes, tal es el caso de la alcaldía nicaragüense que promueve campañas de siembra de árboles, capacita a los jóvenes de cuarto y quinto grados en educación ambiental. En el caso beliceño, existen programas del Ministerio de Recursos Naturales y algunas ONG -como Aububon Belize- los cuales buscan despertar, en la población estudiantil, una conciencia que le permita identificarse con la problemática ambiental tanto a nivel general (mundial), como a nivel especifico (medio donde vive).

La última instancia señalada pretende identificar las relaciones de interacción e independencia presentes en el entorno. Realiza una campaña fuerte con planificación de acciones de capacitación para todo el año. Abarca temas relevantes desde leyes del medio ambiente, leyes de los municipios que respaldan y comprometen a las instituciones del Estado a realizar acciones dirigidas a la protección del medio ambiente y unir esfuerzos con otras entidades públicas. Muy importante es que enfocan el ecosistema al desarrollo sustentable y a no contaminar el medio ambiente promoviendo el reúso de desechos sólidos. Estos programas fortalecen el currículo y, por ende, la formación integral del estudiantado.

Por eso consideramos de suma importancia abrir espacios de diálogo y conectar organizaciones para implementar dichos proyectos o iniciativas en nivel local, nacional y regional.

Los problemas cotidianos, tanto en Belice como en Nicaragua, se abordan mediante la utilización de las disciplinas interrelacionadas y en función de concretar alternativas de solución.

La mayoría de la indiferencia, apatía, y crueldad que vemos, tiene su origen en la falsa educación que damos a nuestros niños acerca de los derechos de los animales, y de su deber con ellos.

J. Todd Ferrier 


\section{Propuestas para lograr un ciudadanía planetaria}

El cambio de actitud y aptitudes tanto del personal docente en su lucha formadora de la nueva generación, como de los chavalos y chavalas, depende mucho de la pedagogía que practicamos desde la escuela. Por eso proponemos:

1. Metodologías constructivistas. En busca de la construcción de un estudiante investigador, se promueven estrategias de aprendizajes y enseñanzas donde se investigue, observe, analice y comunique conocimientos para el desarrollo integral.

2. Una filosofía educativa unificadora. Esta promueve el educado como una persona parte de la comunidad y con la comunidad. De esta manera se transforma el sistema educativo en un buen vivir.

3. La practica de un enfoque interdisciplinario. En este se pretende preparar a la estudiante o al estudiante para la vida, como artífice de su propio aprendizaje a partir de sus experiencias previas, la formación de valores, actitudes, aptitudes, hábitos, habilidades y destrezas que le permitan vivir en armonía con el medio que le rodea.

4. Nuevas formas de acceso a la información y la tecnología (TIC). Con el nuevo milenio es imperativo considera esto como una opción, así, la educación ambiental en las zonas rurales de Centroamérica retoma la oportunidad de revitalizar las metodologías de aprendizajes.

5. Una participación multidireccional. El propósito es lograr una comunicación abierta entre toda la comunidad educativa y luchar por una educación ambiental rural que atienda las desigualdades y exclusiones sociales.

No será posible dar respuesta a los complejos problemas ambientales ni revertir sus causas, sin transformar el sistema de conocimientos que conforman la actual racionalidad social que los genera.

Enrique Leff 
URL: http://www.una.ac.cr/educare

CORREO: educare@una.cr

\section{Conclusiones}

Desde la necesidad, importancia y derecho de una educación ambiental rural contextualizada, que atienda los derechos de la humanidad, se concluye que los currículos básicos y programas en construcción y acción deben atender estas necesidades con metodologías adecuadas que logren los objetivos en forma integral.

El tema de la educación ambiental, en particular en nuestras zonas rurales de Centroamérica, es emergente y está en busca de un desarrollo sostenible en equilibrio con los cambios y transformaciones de la globalización. Por lo tanto, se debe comenzar, desde las bases, la organización comunitaria para hacerle frente a los efectos depredadores de la globalización. Sin duda alguna, uniendo esfuerzos encontraremos el bienestar común.

Tanto el currículo nacional básico de Nicaragua como el de Belice tienen enfoques ambientalistas con principios interrelacionados, con miradas integrales, interdisciplinarias y holísticas en su desarrollo, esto es, todo está escrito; pero hace falta concretarlo en la realidad y evidenciarlo en el buen vivir. En esta tarea la labor del personal docente es imprescindible e idónea, por su capacidad de discernir las consecuencias de la depredación ambiental y porque representan un modelo para la sociedad, con su tacto metodológico. Cabe señalar que, ante todo, el docente requiere de una educación continua actualizada y esta se puede lograr si este profesional percibe el apoyo de todas las instituciones y de la misma sociedad.

Promovamos la información de lo que ocurre en el planeta y en nuestro país; este constante aprendizaje abrirá caminos para una nueva cultura con salud mental y física.

Por lo tanto, la escuela -formadora de la futura generación- debe iniciar, ya, iniciativas ambientales, para forjar una niñez y juventud con mentes creativas, solidarias, con actitudes de respeto a todo ser vivo que forma parte del ciclo de vida.

La educación ambiental será realidad cuando velemos por la salud de la tierra y la protejamos todos juntos -maestros, estudiantes, padres de familia, instituciones, gubernamentales y no gubernamentales, organizaciones sociales-, cuando compartamos las mismas responsabilidades. 


\section{Referencias}

Belize Audubon Society. (2008). An Environmental Agenda for Belize 2008-2013. Full Report [Una agenda ambiental para Belice 2008-2013 Informe completo]. Belize: Autor. Recuperado de http://www.belizeaudubon.org/documents/publications/EnvAgenda-FullReport-web. pdf

División General de Currículo y Desarrollo Tecnológico. Managua, Nicaragua. (2009). Currículo nacional básico. Diseño curricular del subsistema de la educación básica y media nicaragüense. Recuperado de http://www.nicaraguaeduca.edu.ni/uploads/DisenoCurricular.pdf

Gadotti, M. (2001). Pedagogía de la Tierra y cultura de la sustentabilidad. Petrópolis, Recuperado de http://www.rebelion.org/hemeroteca/ecologia/pedagogia251001.htm

Ortega, N. y Velasco, E. (2006). Manual de educación ambiental para escuelas primarias del estado de Guanajuato. Mexico: Fundación de Apoyo Infantil e Instituto de Ecología del Estado.

Solis, V., Madrigal, P., Borrasé, Á y Coto, P. (1998). Ley no. 7786 educación participativa sobre la gente y la naturaleza la Ley de biodiversidad de Costa Rica. San José: Costa Rica: UICN. Recuperado de http://www.metabase.net/docs/uicn/00651.html

\section{Cómo citar este artículo en APA:}

Martínez, M. I. y Carballo, L. (2013). La educación ambiental rural desde las escuelas básicas y por estas. Revista Electrónica Educare, 17(2), 69-79. Recuperado de http://www. revistas.una.ac.cr/index.php/EDUCARE/issue/current

Nota: Para citar este artículo en otros sistemas puede consultar el hipervínculo "Como citar el artículo" en la barra derecha de nuestro sitio web:

http://www.revistas.una.ac.cr/index.php/EDUCARE/index 